\title{
Predictions of the Dependence on the Opposed Flow Characteristics of the Flame Spread Rate over Thick Solid Fuel
}

\author{
C. DI BLASI, S. CRESCITELLI, and G. RUSSO \\ Dipartimento di Ingegneria Chimica \\ Università di Napoli \\ Piazzale V. Tecchio \\ 80125 Napoli, Italy
}

\author{
A. C. FERNANDEZ-PELLO \\ Department of Mechanical Engineering \\ University of California \\ Berkeley, California 94720, USA
}

ABSTRACT

A theoretical model is developed of the effect on the spread of flames over the surface of a thick solid combustible, of the velocity and oxygen concentration of a gas flow opposing the direction of the spread. In the analysis the transient, reactive, gas phase balance equations of energy and species coupled at the interface to the solid phase energy equation are solved numerically to predict the flame spread rate and flame structure dependence on the characteristics of the flow. The calculations for PMMA agree with previous experimental results predicting a spread rate that, for a fixed oxygen concentration, first increases, reaches a maximum and then decreases as the flow velocity is increased. The analysis shows that this behavior is the result of the interaction of two controlling mechanisms: a flame to fuel heet transfer mechanism that dominates at low flow velocities and/or high oxygen concentrations, and a gas phase chemical kinetics mechanism that dominates at high velocities and/or loy oxygen concentrations.

\section{INTRODUCTION}

Experimental studies conducted during the past few years on the process of flame spread over solid combustibles have resulted in a considerable new understanding of its controlling mechanisms [1]. Particularly significant were the studies of the dependence of the flame spread rate on the velocity and oxygen concentration of an opposed gas flow [2] and on the buoyancy and ambient oxygen concentration $[3,4]$. Through phenomenological arguments and semi-empirical nondimensional correlations of these measurements, the above authors postulated that the opposed flow flame spread rate is controlled by the interaction between processes dominated by heat transfer from the flame to the solid and by gas phase chemical kinetics. In the heat transfer dominated regime the spread rate increases with the flow velocity, and in the chemically controlled regime it decreases. The transition from one to the other regime is characterized by a weak dependence of the spread rate on the flow velocity.

The theoretical predictions of these and other experimental flame spread observations have been, to date, only moderately successful due to the complexity of the problem [5]. Most of the flame spread models are thermal models, and consequently only applicable when the reaction 
rate is infinitely fast. The analyses of Ref. [6-9] in particular seem to predict the flame spread rate dependence on the flow properties well, at least qualitatively, in the heat transfer dominated regime. The models that predict best the chemically controlled regime are all numerical analyses. Notable are the analyses of Ref. [10] for flame spread over a thermally thin fuel and of Ref. [11] for a thermaliy thick fuel. The analyses, however, have been employed to simulate flame spread for low values of the oxygen concentration and consequently only the behavior of the spread rate for the chemically controlled regime is predicted. The present work extends the analysis of Ref. [10] to describe the flame spread behavior in both the thermaliy and the chemically controlled regimes.

MODEI

The problem considered is that of a flame spreading over the surface of a thick solid combustible in an oxidizing gas flow opposing the direction of flame propagation. The gas phase reaction is modeled using a one-step second order Arrhenius rate so that finite rate chemical. kinetics effects can be described at least qualitatively. The solid phase heat transfer processes are modeled through the energy equation in its non-reactive, constant properties form. The pyrolysis of the fuel is assumed to be a surface process and to behave as a zero order Arrhenius reaction. Thus, the common assumption of constant gasification temperature is not needed here. No surface oxidation reaction [12], or radiation to or from the surface are considered in the model, and regression of the pyrolyzing surface is neglected.

The mathematical solution of the problem is further simplified by prescribing the flow field, neglecting buoyancy and assuming constant gas properties and pressure. This eliminates the need for using the momentum conservation equations which complicate the mathematical solution of the problem significantly. The velocity profile assigned in this work is that for a Hagen-Poiseuille flow in a channel, one of whose walls is made by the solid fuel. Because of these simplifications, the description of the flame structure, particularly near its leading edge and everywhere at short times after ignition, can only be considered as qualitative. Comparison of the descriptions of the flame structure obtained with this analysis with those obtained with more complicated analyses that do not use the above simplifications $[13,14]$ show that the present analysis describes qualitatively well the flame structure although there are some important localized differences. An important effect not described here is the generation by gas expansion effects of a region of elevated pressure upstream from the flame leading edge [13]. This causes the outward deflection of the flow near the flame front, and consequently the effective reduct $\perp$ on of the gas velocity encountered by the flame at its leading edge. Thus, for the same gas velocity at the flame leading edge, the actual velocity profile will have a larger maximum velocity than that of the corresponding Hagen-Poiseuille profile. Another effect not considered in the model is the flame induced buoyancy flow. This effect is particularly important at low flow velocities where the velocity of the induced flow can be even larger than that of the pure forced flow. Thus the predictions of the present model for very low flow velocity, can not be compared with experimental results unless they are obtained in a microgravity environment. Finally, the neglection of surface radiation should affect the predictive capabili- 
ties of the model at near extinction conditions since surface radiation is a possible cause for extinction.

With the above assumptions, the governing equations that describe the problem are the transient, reactive gas phase energy and species conservation equations and the trensient, inert, solid phase energy equation. With the coordinate system fixed to the solid these equations are:

$$
\begin{aligned}
& \rho\left[\partial Y_{i} / \partial t+u \partial Y_{i} / \partial x\right]=w \mu_{i}+D_{g}\left[\partial^{2} Y_{i} / \partial x^{2}+\partial^{2} Y_{i} / \partial y^{2}\right] \\
& \sum_{i}=i \quad i=I, P, o, F \\
& c_{p} \rho_{g}\left[\partial T_{g} / \partial t+u \partial T_{g} / \partial x\right]=w \Delta H+k_{g}\left[\partial^{2} T_{g} / \partial x^{2}+\partial^{2} T_{g} / \partial y^{2}\right] \\
& c_{s} \rho_{s}\left[\partial T_{S} / \partial t\right]=k_{s}\left[\partial^{2} T_{s} / \partial x^{2}+\partial^{2} T_{s} / \partial y^{2}\right]
\end{aligned}
$$

where $w=-A_{g} \exp \left(-E_{g} / R T_{g}\right) Y_{O} Y_{F} \rho_{g}^{2}$ and $\mu_{i}=M_{i} \nu_{i} / M_{F} \nu_{F} i=I, O, F, P$. The initial conditions are that at $t=0, T_{g}=T_{S}=T_{O}, Y_{F}=Y_{P}=0$, $Y_{O}=Y_{O O}$ and $Y_{I}=Y_{T O}$. The boundary conditions for these equations are: $T_{G}=T_{O}, Y_{O}=Y_{O O}, Y_{F}=Y_{P}=0, Y_{I}=Y_{I O}$ (at the inflow); $\partial T_{g} / \partial x=0$, $\partial^{B} Y_{i} / \partial x=0$ for $i=I, P, O, F$ (at the outflow); $\partial T_{g} / \partial y=0$; $\partial Y_{i} / \partial y=0$ for $i=I, P, O, F$ (at the upper wall of the channel); $\rho_{g} D \partial Y_{F} / \partial y=m\left(Y_{F}-1\right) ; \rho_{g} D \partial Y_{i} / \partial y=m\left(Y_{i}\right)$ for $i=I, O, P ;$ $-k_{g} \partial T_{g} / \partial y=-k_{s} \partial T_{s} / \partial y+m L ; T_{g}=T_{s}$ (at the solid-gas interface); and the pyrolysis mass $f$ lux is equal to: $m=A_{S} \exp \left(-E_{S} / R T_{S}\right) \rho_{S}$ *

The initiation of the flame spread process is simulated by imposing a radiant flux over a small portion of the solid surface at its downstream edge. Once the flame spread process is initiated the external radiation is discontinued. The system of non-linear coupled differential equations (I) to (4) with their initial and boundary conditions are solved numerically using the finite difference method reported in [15]. The time steps are based on the low characteristic time of the problem. A step size of $2.510^{-2} \mathrm{~mm}$ from the surface up to $y=1 \mathrm{~mm}$, followed by a linear variation to $9.410^{-1} \mathrm{~mm}$ for $\mathrm{y}=10 \mathrm{~mm}$, together with a step size along the $x$ direction of $1.410^{-1} \mathrm{~mm}$ have been found to be sufficiently small that a further reduction of the step size does not change the computed results.

\section{RESULTS}

The dependence on the opposed flow velocity and oxygen concentration of the rate of flame spread over a PMMA slabs is examined here. The properties used for PMMA are [2]: $c_{\mathrm{s}}=1.46[\mathrm{~kJ} / \mathrm{Kg}-\mathrm{K}]$, $\rho_{\mathrm{s}}=1.1910^{3} \mathrm{Kg} / \mathrm{m}^{3}, \mathrm{k}_{\mathrm{s}}=1.88 \times 10^{-2}[\mathrm{~J} / \mathrm{smk}], \mathrm{L}=1355.6[\mathrm{~kJ} / \mathrm{Kg}]$. For the pyrolysis reaction $A_{S}=1 . E+6[\mathrm{~m} / \mathrm{sec}]$ and $\mathrm{E}_{\mathrm{S}}=125.52[\mathrm{~J} / \mathrm{mole}]$ are in the range of values suggested in [16]. The gas properties are taken as those of air at ambient temperature. The kinetic and thermokinetic 

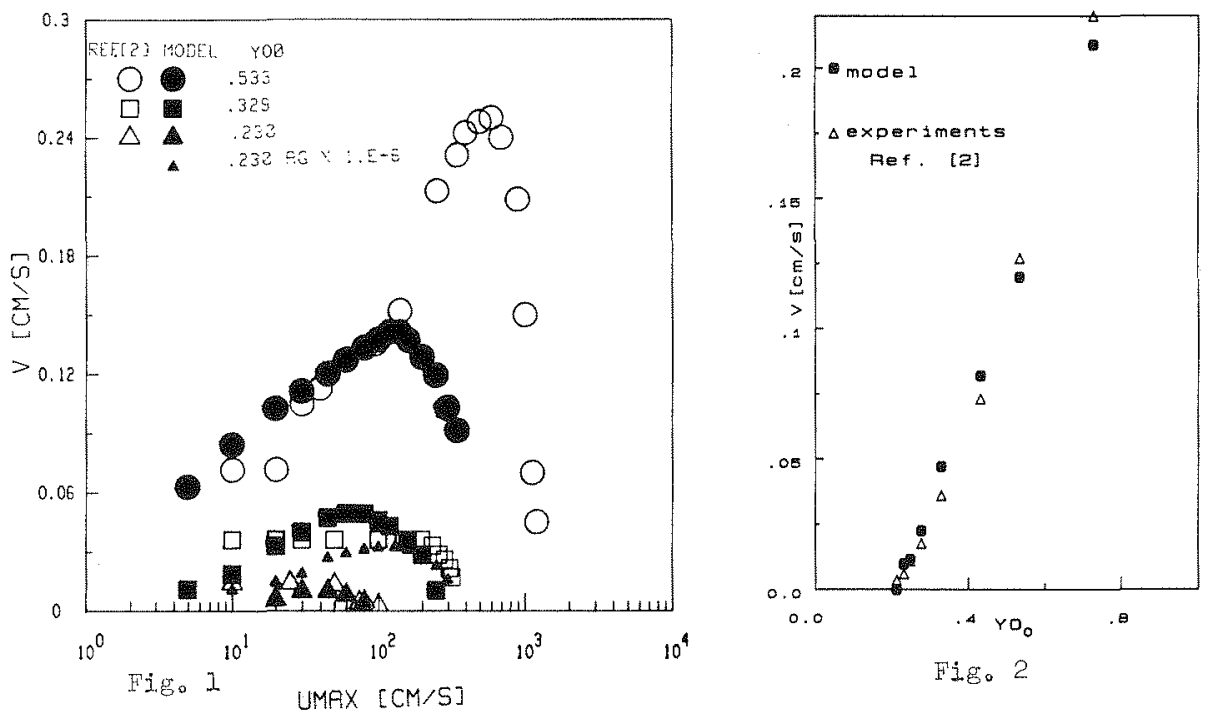

FIGURE 1. Dependence on the opposed flow velocity of the flame spread rate for oxygen mass fraction $0.23,0.329,0.533$. The experiments of Ref. [2] are presented for comparison.

FICURE 2. Dependence of the flame spread rate on the oxygen concentration of the flow for a flow velocity of $45 \mathrm{~cm} / \mathrm{s}$. The experimental meausurements of Ref. [2] are presented for comparison.

constants for the gas phase reaction are [14]: $\mathrm{A}_{\mathrm{g}}=0.16 \mathrm{E}+17\left[\mathrm{Kg} / \mathrm{m}^{3}-\mathrm{sec}\right]$, $\mathrm{E}_{\mathrm{g}}=175.73[\mathrm{~J} / \mathrm{mole}]$ and $\Delta \mathrm{H}=25899[\mathrm{KJ} / \mathrm{Kg}]$. The integration domain is $10 \mathrm{~mm}$ large in the longitudinal direction and $10 \mathrm{~mm}$ and $5 \mathrm{~mm}$ in the norm mal direction in the gas and in the solid respectively. The longitudinal length is more than sufficient for the present calculations because the spread rate is very small and the processes of main interest occur in $1 \mathrm{~mm}$ of the flame front.

Dependence of the Flame Spread Rate on the Flow Characteristics

In Fig. 1 the results are shown for the flame spread rate variation with the flow velocity for oxygen mass fraction of $0.23,0.329$ and 0.533 . For comparison purposes the experimental results reported in Ref. [2] are also included in the figure. It is seen that the analysis describes qualitatively well the experimental results, predicting a spread rate, that for a given oxygen concentration, varies with the flow velocity first increasing and then decreasing as the flow velocity increases. For a given flow velocity the spread rate increases with the oxygen concentration and the location of the maximum is also displaced toward the bigher velocity region as the oxygen concentration increases. The major quantitat $\Rightarrow$ discrepancies between the theory and the experiments occur in thr sry low and very large velocity regions. As it was indicated before is is to be expected given the simplifications introduced in the model. In the experiments, at very low forced flow velo- 
city the flame induced buoyancy flow is dominant, and since for a given oxygen concentration, the induced velocity is approximately constant so is the spread rate $|2|$. The model does not consider buoyancy and consequently this region is not described by the analysis. Instead, a continuosly increasing spreed rate with the velocity is predicted as it is expected to occur in a zero gravity environment.

In the high velocity region where the spread rate decreases as the velocity increases, the spread rate is controlled by gas phase chemical kinetics. Consequently, it is very sensitive to the gas velocity because of the strong dependence of the DamkohIer number on the velocity $[2,4]$. The model, however, does not describe well the velocity field in the vicinity of the flame leading edge. Nor does it describe the deceleration of the velocity in the front of the leading edge of the flame caused by the gas phase expansion in this region [13]. Another factor is that the experiments of Ref. [2] were conducted in a channel $40 \mathrm{~mm}$ deep while the model considers a $10 \mathrm{~mm}$ channel, which for the same maximum flow velocity results in a higher calculated velocity near the wall. The result is that in the analysis the flame leading edge encounters a higher velocity than it would be encountered in experiments performed for the same maximum flow velocity. Consequently, the predicted spread rates are smaller than those measured. This feature of the model can also be interpreted as the analysis giving too much emphasis to the chemical kinetic aspect of the problem, and thus effectively displacing the predicted chemically controlled region toward regions of low gas velocity. This is further corroborated by the results of a case specifically calculated for this purpose with a gas reaction rate pre-exponential factor $1 \mathrm{~s}+6$ time larger. An oxygen mass fraction of 0.23 was used for these calculations and the results are presented in $\mathrm{Fig}$. 1. It is seen that the calculated spread rate variation with the gas velocity follows this trend, thus corroborating the above arguments. Very large differences, at high velocities, are shown for the case of oxygen mass fraction equal to 0.533 . This cannot be attributed only to the assigned flow field but it may be due to the kinetic model employed for the gas phase combustion. First, the present kinetic model is very simple and, second, the data employed for the global reaction have been determined for air and they may not be valied for higher oxygen concentrations.

For intermediate gas flow velocities the flame spread rate is controlled by the transfer of heat from the flame to the solid $[2,4]$ which is less sensitive to the relatively small variations of the velocity profile. Thus in this range of velocities, the analysis should describe quantitatively better the spread of the flame. This is seen from the results presented in Fig. I and most particularly from those of Fig. 2 where the variation of the spread rate with oxygen concentration is presented. The gas velocity selected for these last calculations, $0.45 \mathrm{~m} / \mathrm{sec}$, is comparable to that induced naturally by the flame, and consequently the lowest possible for a meaningful comparison with the experiments. It is seen that the analysis predicts extremely well the variation of the spread rate with the ambient oxygen concentration, again emphasizing the predictive capability of the model.

\section{Controlling Mechanisms of Opposed Flow Flame Spread}

The results of the analysis can also be used to describe the mecham nisms controlling the flame spread process, and thus to verify the phe- 
nomenological arguments used to explain the experimental results in $[1-4]$. The surface heat flux provides information about the transfer of heat from the flame to the solid, and thus it can be used to analyze the thermal. aspects of the flame spread process. It varies along the solid surface and has a maximum in the region where the chemical reaction starts to increase rapidly. The location of this maximum is taken as the flame leading edge location $[10,13]$. The variation of the maximum surface heat flux with the flow velocity is presented in Fig. 3 for the three cases of oxygen concentration considered here. It is seen that for a given flow velocity the heat flux increases with oxygen concentration. This is due primarily to the increase in flame temperature and results in the increase of the flame spread rate with the oxygen concentration. For all oxygen concentrations the heat flux increases with the flow velocity. As it will be shown later, this is due to the flame moving closer to the fuel surface, and to a moderate increase of the flame temperature. The flame temperature increase is the result of a larger fuel concentration at the reaction zone produced by an increase of the gasification rate. The monotonic increase of the heat flux with the flow velocity indicates that the spread rate should increase also monotonically with the opposed flow velocity. This is in agreement with the predictions of the thermal models of flame spread [5] and should be the result obtained with this analysis if the chemical reaction rate is infinite. A partial verification of this aspect of the problem was presented in Fig. 1. through the calculations of the spread rate dependence on the reaction rate premponential factor.

The surface temperature also provides important information about the flame spread process. It can be used to deduce the extent of the solid heated region and by substitution into the pyrolysis rate expression to calculate the gasification rate. The predicted surface temperature is not constant in the pyrolysis zone, instead it increases slightly reaching a maximum near the flame leading edge and then decreases rapidly in front of the flame [11]. The gasification rate follows a similar trend but is magnified because of the exponential dependence of the pyrolysis rate on the surface temperature. The calculations of the variation of the maximum surface temperature with the flow velocity show that the temperature increases with the flow velocity, sharply at first and then slowly for large flow velocities. The surface heat flux follows the same trend. Both the surface temperature and heat flux increase with oxygen concentration. The results agree qualitatively with the experimental results of Ref. [16]. They also confirm that an increase in the velocity and oxygen concentration of the flow results in an increase of the heat transfer from the flame to the fuel and of the gasification rate of the solid combustible. Both effects tend to increase the rate of flame spread.

To explain the mechanisms leading to the decrease of the flame spread rate with the opposed flow velocity it is necessary to analyze the effect of the flow velocity on the flame stmucture, and its interaction with the solid phase. In Fig. 3 the predicted gas and solid phase isotherm fields are presented for a flow with oxygen mass fraction 0.533 and flow velocity $0.20,1.40$ and $3.50 \mathrm{~m} / \mathrm{s}$. The cases with velocities of 0.20 and $3.50 \mathrm{~m} / \mathrm{s}$ have approximately the same spread rate but are respectively in the thermal and chemically controlled regimes (Fig. I). The case with a velocity of $1.40 \mathrm{~m} / \mathrm{s}$ corresponds approximately to the maximum spread rate. For comparison purposes the location of the maximum surface heat flux, identified here as the location of the flame leading edge, is also included in the figure. It is seen that as the 

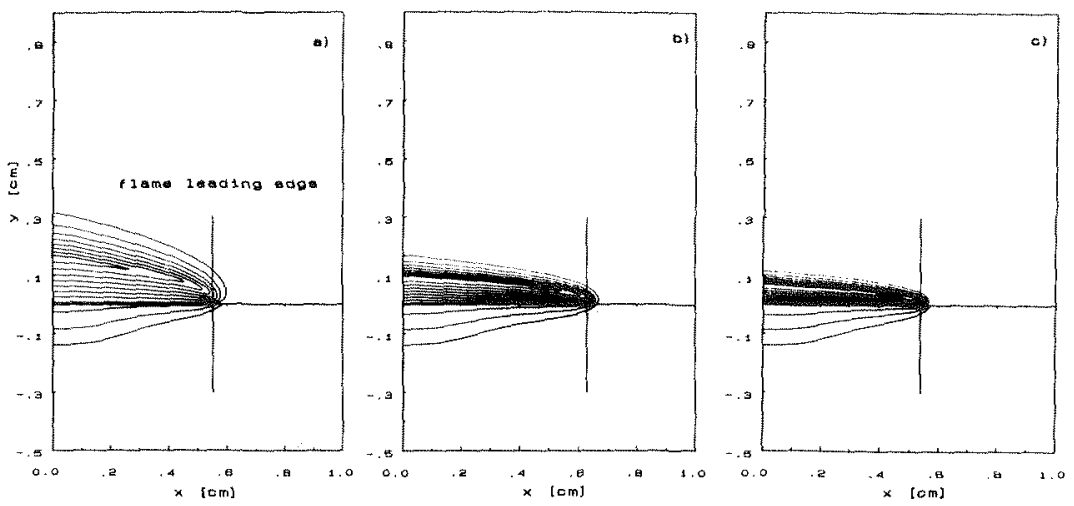

FIGURE 3. Gas and solid phase isotherm field for a flow with oxygen mass fraction of 0.533 and velocity: a) $20 \mathrm{~cm} / \mathrm{s}$, b) $140 \mathrm{~cm} / \mathrm{s}$, c) $350 \mathrm{~cm} / \mathrm{s}$. The external isotherm, for the solid phase, is $400 \mathrm{~K}$ and the increment is $100 \mathrm{~K}$; for the gas phase, the external isotherm is $900 \mathrm{~K}$ and the increment is $600 \mathrm{~K}$.

flow velocity increases the flame moves closer to the surface. This results in larger normal temperature gradients, and consequently heat fluxes, at the solid surface. As the flow velocity is increased the longitudinal temperature gradients also become larger due to the opposition of the flow to the upstream conduction of heat by the flame. With regard to the slowing effect of the flow on the flame spread, it is more informative to compare the relative positions of the gas and solid phases thermal Layers and the overall structure of the flame at its leading edge. From the results presented in Fig. 4 it is seen that as the flow velocity is increased the upstream edge of the gas thermal layer recedes with respect to the corresponding edge in the solid layer. Thus, the effect is basically that of the flow pushing back the flame, 1.e., whether the flame advances or recedes depends on the relative magnitude between the burning velocity and the flow velocity [18].

This concept can also be interpreted in terms of the ratio of the flow to the chemical time, i.e., the Damkohler number. The flow time is given in this case by the ratio of the heated length ahead of the flame to the flow velocity. Referring again to Fig. 4, it is seen that the upstream heated length decreases as the velocity increases, which results in the flow time decreasing strongly with the flow velocity. The chemical time, which is inversely proportional to the reaction rate, decreases because of the increase in the fuel concentration with the flow velocity, but only silightly. The result is a decrease of the Damkohler number with the flow velocity which leads to a decrease in the spread rate. The decrease of the Damkohler number can be clearly seen from the fuel and oxygen concentration profiles presented in Fig. 4. Observation of the concentration profile upstream from the flame leading edge shows that a reduction in the Damkohler number results in the leakage of fuel through the reaction and its diffusion upstream. Whether this decreasing effect of the flow on the chemical reaction will affect the spread rate or not depends on the relative magnitude of the chemical and flow time. If the chemical time is much smaller than the flow time, the spread of the flame will be controlled by the rate of 


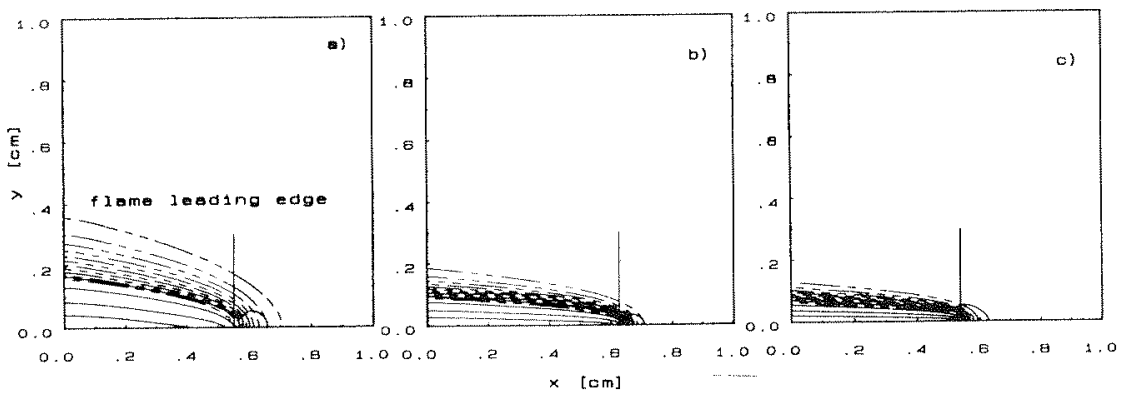

FIGURE 4. Constant concentration lines of fuel and oxygen for a flow with oxygen concentration of 0.533 and velocity: a) $20 \mathrm{~cm} / \mathrm{s}$, b) $140 \mathrm{~cm} / \mathrm{s}$, c) $350 \mathrm{~cm} / \mathrm{s}$. The isolines for the fuel mass fraction correspond to $0.5,0.3,0.1,0.001$; for the oxygen mass fraction the internal line is 0.005 and the increment is 0.05 .

fuel gasification, i.e., by the heat transfer from the flame to the fuel. On the other hand, if the chemical time is of the same order as the flow time, the spread of the flame will be controlled by the rate at which the fuel can be consumed, i.e., by chemical kinetics.

This is further verified by the comparison of the isotherm fields presented in Fig. 5 for the two cases at the same flow velocities 10.80 $\mathrm{m} / \mathrm{s}$ ) but oxygen mass fraction of 0.533 and 0.23 . The former one is in the thermally controlled regime while the latter is in the chemically controlled regime (Fig. 1). The reaction rate for the 0.533 oxygen mass fraction is of the order of ten times larger than that for 0.23 mass fraction, and as it can be seen from the results of $\mathrm{Fig}$. 5, in the high concentration case the gas thermal layer leading edge is approximately at the same position as the corresponding point in the solid. On the other hand for the 0.23 mass fraction case, the gas thermal layer leading edge is behind that in the solid.
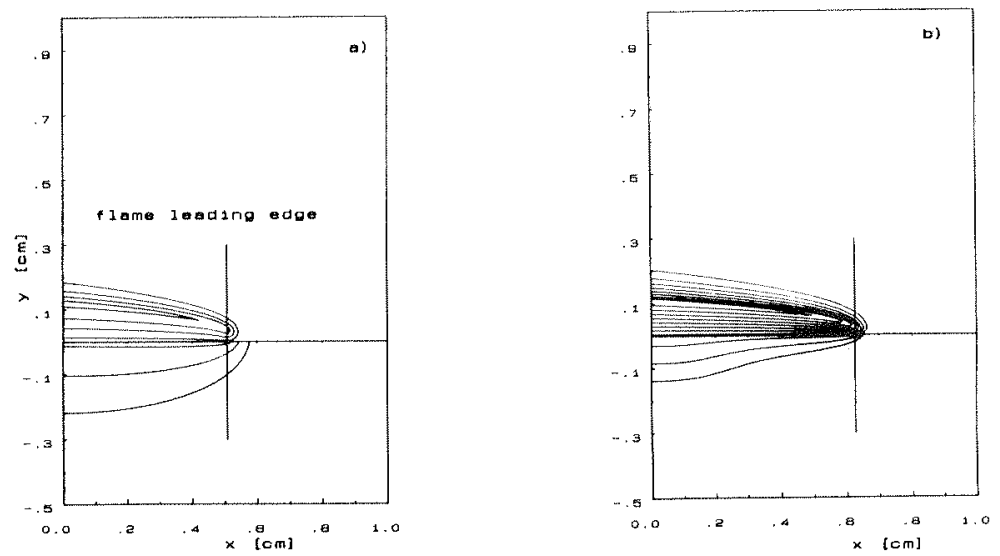

FIGURE 5. Gas and solid phase isotherm field for a flow velocity of $80 \mathrm{~cm} / \mathrm{s}$ and oxygen mass fraction of: a) 0.23 and b) 0.533 . The external isotherm, for the solid phase, is $400 \mathrm{~K}$ and the increment is $100 \mathrm{~K}$; for the gas phase, the external isotherm is $900 \mathrm{~K}$ and the increment is $600 \mathrm{~K}$. 


\section{CONCLUDING REMARKS}

The analysis developed in this work of the spread of flame a over the surface of a thick solid combustible in an opposed forced flow represents an important step forward in the modeling of the flame spread process. The results of the analysis show a complex interaction between gas phase chemical kinetic mechanisms and flame to solid heat transfer processes. Through the predictions of the flame structure, the solid thermal field and the interface variables it was possible to theoretically verify the phenomenological arguments and semi-empirical correlations used to explain the nature of the experimental measurements and the controlling mechanisms of the process. The complete solution of the problem, however, is too complicated and may not be achieved in the immediate future. It should include, among others, the detailed analysis of the flow field, buoyancy, flame and surface radiation, multiple reactions chemical kinetics, turbulence, material charring or in depth pyrolysis. The inclusion of each one of these processes introduces major complexities to the solution of the problem. The results of this analysis, however, are most encouraging and indicate the direction to follow in order to completely solve the problem.

\section{ACKNOWLEDGMENTS}

This work was supported by the Progetto Finalizzato Chimica Fine e Secondaria of C.N.R., Italy. The last author (A.C. Fernandez-Pello) was on visit at the Dipartimento di Ingegneria Chimica, Università di Napoli, Italy, during the development of this work. His work was supported by the University of Naples and by the National Bureau of Standards, U.S.A., under grant No. NB83NADAl020.

\section{NOMENCLATURE}

A pre-exponential factor in gas phase reaction

$A_{s}^{g} \quad$ premexponential factor in pyrolysis reaction

$c_{p}$ gas phase specific heat at constant pressure

$c_{s}$ solid phase specific heat

D gas phase diffusion coefficient

$\mathrm{E}_{g}$ activation energy for gas phase reaction

$\mathrm{E}_{\mathrm{S}}^{\mathrm{G}} \quad$ activation energy for pyrolysis reaction

$\Delta \mathrm{H}$ heat of combustion per unit mass of fuel

k gas phase thermi conductivity

$k_{s}^{g}$ solid phase thermal conductivity

$\mathrm{I}^{\mathrm{s}}$ heat of pyrolysis

m pyrolysis mass flux

$T$ gas phase temperature

$T_{s}^{g} \quad$ solid phase temperature

$t$ time

u gas velocity

umax maximum gas velocity

$\checkmark$ llame spread rate

$x$ coordinate parallel to the fuel surface

$y$ coordinate normal to the fuel surface 


$\begin{array}{ll}Y & \text { mass fraction species } i \\ v_{i} & \text { stoichiometric coefficient } \\ { }_{\mathrm{o}} & \text { gas phase density } \\ \rho_{s} & \text { solid phase density } \\ \text { Subscripts } \\ \text { F } & \text { fuel } \\ g & \text { gas phase } \\ \text { I } & \text { inert } \\ \text { i } & \text { species } \\ 0 & \text { oxidant } \\ \text { P } & \text { product } \\ \text { s } & \text { solid phase } \\ 0 & \text { ambient conditions }\end{array}$

\section{REFERENCES}

1. Fernandez-Pe110, A. C., and Hirano, T., Combustion Science and Technology 32, p. 1 (1983).

2. Fernandez-Pello, A. C., Ray, S., and Glassman, I., Eighteenth Symposium (Int.) on Combustion, The Combustion Institute, p. 579 (1981).

3. Altenkirch, R. A., Eichorn, R., and Shang, P. C., Combustion and Flame 37 , p. 71 (1980).

4. Altenkirch, R. A., Eichorn, R., and Rizvi, A. R., Combustion Science and Technology 32 , p. 49 (1983).

5. Fernandez-Pel1o, A. C., Combustion Science and Technology 39, p. 119 $(1984)$.

6. de Ris, J. N., Twelfth Symposium (Int.) on Combustion, The Combustion Institute, Pittsburgh, PA, p. 241 (1969).

7. Wichman, I. S., Williams, F. A., and Glassman, I., Nineteenth Symposium (Int.) on Combustion, The Combustion Institute, p. 835 (1982).

8. Wichman, I. S., and Williams, F. A., Combustion Science and Technology 32, p. 91 (1983).

9. Wichman, T. S., Combustion and Flame 50, p. 287 (1983).

10. Frey, A. E., Jr., and T'ien, J. S., Combustion and Flame 26, p. 257 $(1976)$.

11. Di Blasi, C., Continilio, G., Crescitelli, S., and Russo, G., Combustion Science and Technology, (in press) (1987).

12. Kashiwagi, T., and ohlemiller, T. J., Nineteenth Symposium (Int.) on Combustion, the Combustion Institute, p. 815 (1982).

13. Nao, C. P., Kodama, H., and Fernandez-Pello, A. C., Combustion and Flame 57, p. $209(1984)$.

14. Kodama, H., Miyasaka, K., and Fernandez-Pello, A. C., Combustion Science and Technology (in press) (1987).

15. Di Blasi, C., and Continilio, G., Proceedings of the Chemical Engineering Fundamentals, XVIII Congress, p. 361 (1987).

16. Lengelle, G., AIAA Journal Vol. 8, No. 11, p. 1989 (1970).

17. Jellinek, H. H. G., ed. Degradation and Stabilization of Polymers, A Series of Comprehensive Reviews, Mlsevier, Vol. 1, p. 310 (1983).

18. Ray, S. R., and Glassman, I., Combustion Science and Technology 32 , p. $33(1983)$. 